\title{
THE PHYSICAL PICTURE OF BEAM LOADING IN THE BLOWOUT REGIME*
}

\author{
M. Tzoufras ${ }^{\dagger}$, W. Lu, F. S. Tsung, C. Huang, W. B. Mori, UCLA, CA 90095, USA \\ J. Vieira, R. A. Fonseca, L. O. Silva, GoLP, IST, 1049-001 Lisboa, Portugal
}

\begin{abstract}
The feasibility of Laser/Plasma Wakefield Accelerators depends upon the ability to efficiently load a bunch of electrons to the wakefield driven by a laser or an electron beam propagating through a plasma. Such accelerators are expected to operate in a nonlinear regime -the blowout regime-, where the driver is intense enough to push all plasma electrons aside. Since the properties of the accelerator are determined by the three dimensional shape of the wakefield in the blowout regime, a description of beam loading must involve the interaction between this shape and the accelerating electrons. In order for the quality of the accelerating beam to be high, the modification of the plasma structure due to the presence of the beam must be small. Maximizing the beam charge while maintaining its quality high requires tailoring of the beam profile. This tailored profile may then be scaled up for more powerful drivers.
\end{abstract}

\section{INTRODUCTION}

Laser/Plasma Wakefield Accelerators (LWFA/PWFA) depend on the plasma medium to transfer some of the energy of the (Laser or Beam) driver to a bunch of accelerating electrons $[1,2]$. For this to happen, the driver that moves with a velocity very close to the speed of light $v_{g} / c \rightarrow 1^{-}$, constantly "carves" on the plasma "substrate" an accelerating structure, that moves with the same velocity. An electron bunch with velocity $v_{0}, v_{g} \leq v_{0}<c$, that is initially placed at an appropriately chosen spot behind the driver experiences an accelerating force as long as it remains in phase with the accelerating structure. As the energy of the electrons increases they keep approaching the driver until they reach the dephasing point at which the acceleration process terminates. The quality of the resulting electron beam depends on the interaction of the electrons with the wakefields.

The advantage of plasma-based acceleration, compared to conventional acceleration, lies on the fact that the accelerating structure can support enormous accelerating gradients, as plasmas are not bound by material breakdown limits. However, the ability to generate high quality monoenergetic beams similar to those being generated by conventional methods has not been demonstrated yet. Exper-

\footnotetext{
* This work is supported by DOE under Grants No. DE-FG0392ER40727, No. DE-FG52-06NA26195, No. DE-FC02-01ER41179, No. DE-FG02-03ER54721 and NSF under Grant No. NSF-Phy-0321345. The simulations were performed on the Dawson cluster and at NERSC under mp113.

† mtzouf@ucla.edu
}

iments $[4,5]$ and simulations $[6,7,8]$ have shown that electrons originating in the background plasma can enter the accelerating structure and form a quasi-monoenergetic electron beam. Still, for plasma-based acceleration to become an attractive alternative to conventional methods, understanding of the physics that determine the beam quality in LWFA/PWFA is necessary.

\section{THEORETICAL MODELS}

\section{Linear Regime Versus Blowout Regime}

Beam loading in the linear regime of plasma-based acceleration was studied in detail by T. Katsouleas et al. [9]. In this regime the wake is described by a linear P.D.E. that can be directly integrated using Green's functions. The wake of the driver is then superimposed to that caused by the accelerating electrons to yield the final form of the fields. Using this method T. Katsouleas et al. determined the number of electrons that can absorb all of the energy in the wake; found the profile of the beam that makes the accelerating field experienced by all electrons identical; and discussed the issues of emittance and transverse beam loading. It was found that only very narrow electron beams $\sigma_{r} \ll c / \omega_{p}$ can maintain low energy spread and emittance. Such configurations however, may only support beams with a small amount of charge.

Higher charge and higher efficiency can be achieved in the nonlinear blowout regime $[10,11,12]$, where the force by the laser or beam driver is strong enough to expel all plasma electrons generating a pure ion channel. The accelerating force in the ion channel is then independent of the distance $r$ from the axis of the propagation $z$. Thus, the transverse beam loading is not an issue in the blowout regime. In addition to the accelerating force, the ion channel exerts on the accelerating particles a transverse force linear with $r$, which means that the emittance of the electron beam is conserved.

\section{Theoretical Description of the Blowout Regime}

In this nonlinear regime the equations describing the three dimensional wakefield were recently derived by $\mathrm{W}$. $\mathrm{Lu}$ et al. [11, 12]. The shape of the plasma bubble formed behind the driver can be represented by the trajectory of the innermost electron in the plasma sheath surrounding the ion channel. This trajectory $r_{b}(\xi)$ is found in a frame moving with the speed of light towards positive $z$, where $r_{b}$ is the local radius in cylindrical geometry and $\xi=c t-z$ measures the distance from the front of the driver. For blowout 
radius $R_{b} \gg c / \omega_{p}, r_{b}(\xi)$ is the solution of the second order O.D.E.:

$$
r_{b} \frac{d^{2} r_{b}}{d \xi^{2}}+2\left(\frac{d r_{b}}{d \xi}\right)^{2}+1=\frac{4 \lambda(\xi)}{r_{b}^{2}}
$$

where $\lambda(\xi)$ is a term proportional to the charge per unit length of an electron beam $\left(\lambda(\xi)=\int_{0}^{r_{b} \gg \sigma_{r}} r \frac{n_{b}}{n_{p}} d r\right)$ or to the transverse ponderomotive force $\left(\lambda(\xi)=-r_{b}^{2} \frac{d}{d r} \frac{|a|^{2}}{4}\right)$ of a laser at the position $\xi$.

If we use normalized units $\bar{r}_{b} \rightarrow r_{b} / R_{b}, \bar{\xi} \rightarrow \xi / R_{b}$ in Eq. (1) the right hand side becomes $\lambda(\xi) /\left(R_{b}^{2} \bar{r}_{b}^{2}\right)$. Therefore, the physics described by Eq. (1) are independent of scale length as long as $\lambda(\xi) \propto R_{b}^{2}$. If $\lambda(\xi)$ corresponds to the beam loaded electron bunch the number of particles in it becomes: $N=n_{p} \int_{-\infty}^{\infty} 2 \pi \lambda(\xi) d \xi=$ $n_{p} R_{b} \int_{-\infty}^{\infty} 2 \pi \lambda(\xi) d \bar{\xi}=n_{p} R_{b}^{3} \times C_{Q}$ where $C_{Q}$ is a constant that depends on the normalized bunch profile. The number of particles in the bunch scales as:

$$
N \propto n_{p} R_{b}^{3}
$$

For a laser driver the matching condition [10]: $k_{p} R_{b} \simeq$ $2 \sqrt{a_{0}} \simeq 2 \sqrt{2\left(\frac{P}{P_{c}}\right)^{1 / 3}}$ can be substituted in Eq. yield:

$$
N \simeq C_{Q} n_{p} k_{p}^{-3} \sqrt{\frac{P}{P_{c}}} \simeq \frac{C_{Q}}{k_{0} r_{e}} \sqrt{\frac{P}{P_{r}}} \propto \frac{1}{k_{0}} \sqrt{\frac{P}{P_{r}}}
$$

where $P$ is the laser power, $P_{c}=2 P_{r} k_{0}^{2} / k_{p}^{2}$ the power for relativistic self-focusing, $P_{r}=m_{e}^{2} c^{5} / e^{2} \simeq 8.5 G W, r_{e}=$ $e^{2} / m c^{2}$ is the classical electron radius, and $k_{0}$ is the laser wavenumber. The scaling (3) is identical to that derived in references $[10,13]$ using energy balance arguments.

For ultraintense drivers $\left(R_{b} \gg c / \omega_{p}\right)$ the trajectory $r_{b}(\xi)$ resembles a circular "bubble". In the back of this structure a bunch of accelerating electrons can be loaded. The beam loaded bunch repels the electrons in the sheath, thereby slowing down their motion toward the z-axis. This motion of the particles in the sheath is intimately related to the accelerating field experienced by the loaded bunch which is $[11,12]$ :

$$
E_{z} \simeq \frac{1}{2} r_{b} \frac{d r_{b}}{d \xi}=\frac{1}{2} r_{b} v_{r b}
$$

If the total charge and the charge per unit length of the loaded bunch are both sufficiently large the electrons in the sheath are forced to reverse their transverse motion. Thus, the sign of the transverse velocity $v_{r b}$ changes, at which point the wakefield $E_{z}$ becomes decelerating. Large variations of $E_{z}$ due to beam loading are undesirable because they result in electron beams with large energy spread. Therefore, beams with low energy spread may only emerge from accelerating structures that do not deviate significantly from the nearly spherical shape of the "bubble" with no beam loaded electrons [11].

\section{Energy Balance}

In [10] W. Lu et al. assumed that the bubble is a sphere and calculated the total electromagnetic energy $\mathcal{E}$ in the fields of the ion channel in the absence of the electron bunch. A similar amount of energy was estimated to exist in the electron sheath, so that the total energy available to the beam loaded bunch within a single nonlinear plasma wavelength is the sum of the field energy and the kinetic energy in the sheath of one bubble, approximately $2 \mathcal{E}$.

This energy, once absorbed by the electron bunch, is transformed to kinetic energy of the accelerating electrons. If the electron bunch contains $N$ particles and experiences an accelerating field equal to $E_{z}(\xi)$, while riding the wake at phase $\xi$, it absorbs energy $N e E_{z}(\xi) 2 R_{b}$ within distance $2 R_{b}$. Equating the kinetic energy added to the electron bunch in each nonlinear plasma wavelength $N e E_{z}(\xi) 2 R_{b}$ with the energy available in the bubble $2 \mathcal{E}$ we find $N e E_{z}(\xi) 2 R_{b} \simeq 2 \mathcal{E}$.

During the entire acceleration process the average wakefield is $\left\langle E_{z}\right\rangle$. Substituting this field instead of $E_{z}(\xi)$ we obtain an estimate for the number of particles:

$$
N e\left\langle E_{z}\right\rangle 2 R_{b} \simeq 2 \mathcal{E} \Rightarrow N=\frac{\mathcal{E}}{R_{b} e\left\langle E_{z}\right\rangle}
$$

Using the estimates for the energy $\mathcal{E} \simeq$ $\frac{1}{60}\left(k_{p} R_{b}\right)^{5}\left(\frac{m^{2} c^{5}}{e^{2} \omega_{p}}\right)$ and average field $\left\langle E_{z}\right\rangle \simeq \frac{1}{4} \frac{m c \omega_{p}}{e} k_{p} R_{b}$ we obtain:

$$
N \simeq \frac{1}{15} \frac{\left(k_{p} R_{b}\right)^{3}}{k_{p} r_{e}}
$$

Using the matching condition for a laser:

$$
N \simeq \frac{8}{15} \frac{1}{k_{0} r_{e}} \sqrt{\frac{P}{P_{r}}}
$$

Thus, using energy balance arguments, the coefficient $C_{Q}$ was estimated in [10] to be $C_{Q} \simeq 8 / 15$.

\section{COMPUTER SIMULATIONS}

The coefficient $8 / 15$ for the scaling of the number of particles (7) is only a rough estimate that was obtained on the basis of numerous crude assumptions. Gordienko and Pukhov [13] measured from their simulations a coefficient close to 1.8 , albeit for an accelerated bunch with significant energy spread and a significantly elongated bubble. This implies that in their case the charge might have been excessive. Here we use particle-in-cell simulations with the code OSIRIS [14] to study beam loading in the blowout regime.

The simulations were performed in cylindrical geometry. A beam driver intense enough to generate a plasma bubble with radius $k_{p} R_{b} \simeq 10$ is used. The beam loaded electron bunch has a Gaussian profile $N_{b}(z)=\frac{N_{b}}{\sqrt{2 \pi} \sigma_{z}} e^{-z^{2} /\left(2 \sigma_{z}^{2}\right)}$. The transverse profile is also Gaussian but it is not shown, since it is irrelevant as long as $\sigma_{r} \ll R_{b}$. The spot size $\sigma_{z}$ of the bunch is $k_{p} \sigma_{z} \simeq 1$. The simulations were performed 

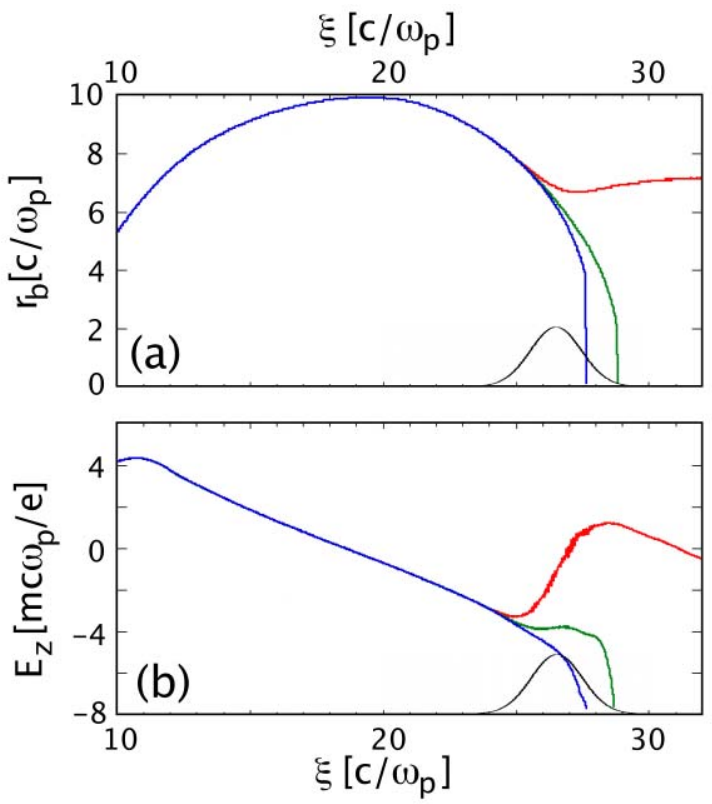

Figure 1: (a) The trajectory of the innermost electron is shown for beamloading with constant $C_{Q}=\frac{1}{6}$ (blue line), for $C_{Q}=\frac{1}{2}$ (green line) and for $C_{Q}=\frac{3}{2}$ (red line). The location of the beam loaded electron bunch is shown with the black line. (b) A lineout of the wakefield $E_{z}$ is presented for beam loading with $C_{Q}=\frac{1}{6}$ (blue line), $C_{Q}=\frac{1}{2}$ (green line) and for $C_{Q}=\frac{3}{2}$ (red line).

in a $50 \frac{c}{\omega_{p}} \times 40 \frac{c}{\omega_{p}}$ moving window, traveling with the speed of light so as to follow the accelerating structure.

In Figure 1(a) the shape of the bubble is shown for three different cases of beam loading, one with $C_{Q}=\frac{1}{6}$ corresponding to approximately $1 / 3$ of the charge predicted by (7), one with $C_{Q}=\frac{1}{2}$ which is approximately the charge predicted by (7) and one with $C_{Q}=\frac{3}{2}$ which is close to the number measured in [13]. In Figure 1(b) we present the corresponding wakefield for the three cases. For convenience the bunch density profile has been sketched in both (a) and (b) with a black line.

From Figure 1(a) it is clear that the bubble for the case $C_{Q}=\frac{1}{2}$ is only slighted elongated compared to the case $C_{Q}=\frac{1}{6}$, which means that the wakefield may be appropriate for accelerating high quality beams. This is verified by Figure 1(b), where the accelerating field (green line) for the case $C_{Q}=\frac{1}{2}$ is nearly constant in the vicinity of the electron bunch. On the contrary for $C_{Q}=\frac{3}{2}$ the beam loading is severe enough to make the electrons in the sheath reverse the direction of their transverse motion and start moving away from the $\xi$ axis. The shape of the ion channel is then (red line in Figure 1(a)) severly modified and the corresponding wakefield (red line in Figure 1(b)) crosses 0 and becomes decelerating for part of the bunch. Such a wakefield leads to unacceptably large energy spread of the accelerating beam.

\section{CONCLUSIONS}

Beam loading of a LWFA/PWFA in the nonlinear blowout regime, where high efficiency and beam quality can both be achieved, has been discussed. The scaling of the number of particles that can be loaded has been derived (in agreement with References $[10,13]$ ) and the constant in front of the scaling is discussed. Simulations for untailored (Gaussian) bunches indicate that high beam quality cannot be achieved, unless the charge is low enough to not cause severe distortion of the shape of the bubble. The constant in the scaling is then close to the rough estimate obtained by [10].

\section{REFERENCES}

[1] T. Tajima and J.M. Dawson, Phys. Rev. Lett. 43, 267 (1979).

[2] P. Chen et al, Phys. Rev. Lett., 54, 693 (1985).

[3] J. B. Rosenzweig et al, Phys. Rev. A 44, R6189 (1991).

[4] S. P. D. Mangles et al., Nature 431, 535 (2004); C.G.R Geddes et al., Nature 431, 538 (2004); J. Faure et al., Nature, 431, 541 (2004).

[5] W. P. Leemans et al, Nature Phys. 2, 696 (2006).

[6] A. Pukhov and J. Meyer-ter-vehn, Appl. Phys. B: Lasers Opt. 74, 355 (2002).

[7] F.S.Tsung et al, Phys. Rev. Lett., 93, 185002 (2004).

[8] F.S.Tsung et al, Phys. Plasmas 13, 056708 (2006).

[9] T. Katsouleas et al, Particle Accelerators, 1987, Vol. 22, pp 81-99.

[10] W. Lu et al, Phys. Rev. ST Accel. Beams 10, 061301 (2007)

[11] W. Lu et al, Phys. Rev. Lett. 96, 165002 (2006).

[12] W. Lu et al, Phys. Plasmas 13, 056709 (2006).

[13] S.Gordienko and A. Pukhov, Phys. Plasmas 12, 043109 (2005).

[14] R. G. Hemker et al., Proceedings of the 1999 Particle Accelerator Conference, edited by A. Luccio and W. Mackay (IEEE, Piscataway, NJ, 1999), Vol. 5, p. 3672; R. Fonseca et al., Lecture Notes in Computer Science (Springer, Heidelberg, 2002), Vol. 2331, p. 342. 